\title{
Eudaimonic Modeling of Moviegoers
}

\author{
Marko Tkalčič \\ Faculty of Computer Science \\ Free University of Bozen-Bolzano \\ Bozen-Bolzano, Italy \\ marko.tkalcic@unibz.it
}

\author{
Bruce Ferwerda \\ Department of Computer Science and Informatics \\ School of Engineering \\ Jönköping University \\ Jönköping, Sweden \\ bruce.ferwerda@ju.se
}

\begin{abstract}
One of the important aspects of movie-making is to trigger emotional responses in viewers. These emotional experiences can be divided into hedonic and eudaimonic. While the former are characterized as plain enjoyment, the latter deal with getting greater insight, self-reflection or contemplation. So far, modeling of user preferences about movies and personalization algorithms have largely ignored the eudaimonic aspect of the consumption of movies. In this paper we fill this gap by exploring what are the relationship between (i) eudaimonic and hedonic characteristics of movies, (ii) users' preferences and (iii) users' personality. Our results show that eudaimonic user profiling effectively divides users into pleasureseekers and meaning-seekers.
\end{abstract}

\section{CCS CONCEPTS}

- Human-centered computing $\rightarrow$ User models; User studies;

\section{KEYWORDS}

eudaimonia, movie preferences, user model

\section{ACM Reference Format:}

Marko Tkalčič and Bruce Ferwerda. 1997. Eudaimonic Modeling of Moviegoers. In Proceedings of ACM Woodstock conference (WOODSTOCK'97), Jennifer B. Sartor, Theo D'Hondt, and Wolfgang De Meuter (Eds.). ACM, New York, NY, USA, Article 4, 5 pages. https://doi.org/10.475/123_4

\section{INTRODUCTION}

Recent research on user feedback in personalized systems, such as recommender systems, is focusing on how much a user likes an item. For example, in Facebook, users can rate a post using the like button. However, a single like or rating on a numeric scale does not capture the whole experience. Furthermore, the experience of consumption of an item (listening to a song, watching a movie) does not have only hedonic qualities (fun, enjoyment, relaxation) but also eudaimonic qualities, which are related to meaning and purpose [8].

A general research direction related to eudaimonic qualities of experience of content consumption we are pursuing is to use eudaimonia for personalization. The assumption is that users differ in their need for eudaimonic experiences, i.e. some people prefer

Permission to make digital or hard copies of part or all of this work for personal or classroom use is granted without fee provided that copies are not made or distributed for profit or commercial advantage and that copies bear this notice and the full citation on the first page. Copyrights for third-party components of this work must be honored.

For all other uses, contact the owner/author(s).

WOODSTOCK'97, fuly 1997, El Paso, Texas USA

(c) 2016 Copyright held by the owner/author(s)

ACM ISBN 123-4567-24-567/08/06 .. \$15.00

https://doi.org/10.475/123_4 to just have fun, while other people may prefer to spend their time contemplating meaning and purpose. Similarly, content differs in the experience quality they induce. For example, the movies The Hangover and La vita e' bella are both comedies. But while the former is a shallow comedy with a series of simple jokes the latter deals with deeper issues, such as the holocaust.

In order to devise personalization approaches using eudaimonia there are a lot of steps to make, since it is an unexplored area. We foresee the following steps need to be taken: (i) unobtrusive inference of eudaimonic and hedonic user preferences, (ii) automatic labeling of movies' eudaimonic and hedonic qualities, and (iii) a personalized recommender system that takes advantage of eudaimonic and hedonic features. We aim at kicking-off the research path in this direction with the results reported here. The contributions of this paper are: (i) a characterization of users in terms of eudaimonic and hedonic preferences (Sect. 4.1), (ii) a characterization of movies in terms of their eudaimonic and hedonic qualities (Sect. 4.2) and (iii) a correlation analysis between users' reported eudaimonic and hedonic preferences and their reported personalities (Sect. 4.3).

\section{RELATED WORK}

There is substantial disjoint work in the domains of (i) conceptualizing eudaimonic and hedonic experiences for entertainment and (ii) work covering the relationships between personality and user preferences.

In positive psychology, happiness is often described through two opposite concepts: hedonism and eudaimonism [1]: the hedonic view equates happiness with pleasure, comfort, and enjoyment, whereas the eudaimonic view equates happiness with the human ability to pursue complex goals which are meaningful to the individual and society. Oliver and Raney [9] have carried out research to identify whether there are distinct eudaimonic and hedonic motivations for consuming entertainment. Through a series of studies they devised an instrument for measuring the eudaimonic and hedonic qualities of entertainment experiences. They showed that in addition to viewing movies for purposes of fun and pleasure, individuals also turn to entertainment for purposes of greater insight and meaningfulness. Wirth et al. [13] further extended Oliver's work by analyzing what are the hedonic and eudaimonic qualities of movies with good and bad endings and found significant differences.

Personality traits account for our individual differences. Among several models of personality the most widely used is the Five Factor Model (FFM), which contains the following factors: openness, conscientiousness, extraversion, agreeableness and neuroticism [7]. Several works has shown that personality is related to user 
preferences for entertainment content, such as music $[3,10,12]$ and movies [5].

Little work has been done on relating personality and eudaimonic experiences. One of these is a study using functional magnetic resonance to identify neural pathways linking personality traits and eudaimonic well-being [6].

With this work we aim at bringing together the disjoint work of user modeling, with the intent of personalizing movie recommendations, and eudaimonic characterization of entertainment experiences. We do this by profiling users in terms of eudaimonic and hedonic preferences, characterizing movies in terms of their eudaimonic and hedonic qualities and correlating users' reported eudaimonic and hedonic preferences and their reported personalities.

\section{EXPERIMENT}

In order to perform the analysis we performed a user study to acquire data. We let the subjects choose movies from a pool of popular movies. For the hypothetical context of choosing a movie to watch alone on a Saturday evening, each subject had to choose the most appropriate movie (the liked movie) and the least appropriate movie (the disliked movie). The subjects were then asked to describe, for each of the two movies, their viewing experience in terms of hedonic and eudaimonic experience using an adaptation of the scale developed by [9]. After answering the movie-related questions, the subjects filled in the ten-items personality questionnaire (TIPI).

Table 1: Excerpt of movie titles used in the experiment. The eudaimonic and hedonic qualities are our subjective assessments

\begin{tabular}{lcc}
\hline Title & Eudaimonic & Hedonic \\
\hline Manchester by the sea & $\mathrm{Y}$ & $\mathrm{N}$ \\
Bad Moms & $\mathrm{N}$ & $\mathrm{Y}$ \\
Mad Max: Fury Road & $\mathrm{N}$ & $\mathrm{Y}$ \\
The Shawshank Redemption & $\mathrm{Y}$ & $\mathrm{N}$ \\
Inside Out & $\mathrm{Y}$ & $\mathrm{Y}$ \\
\hline
\end{tabular}

The pool of movies has been hand-picked by the authors by choosing the most popular movies from the years 2015, 2016, 2017, and a subset of all-time most popular movies. This maximized the probability that the subjects had watched at least a couple of movies. We choose to limit the subjects to a limited pool of movies instead of giving them freedom to choose their own movies to assess in order to increase the chance of having overlapping movie evaluations. We manually balanced the pool of movies in such a way that it contained a roughly equal amount of movies that contained eudaimonic qualities (e.g., Manchester by the sea) and not (e.g.. Bad Moms) according to our judgment. We used the IMDB most popular movies list as the source ${ }^{1}$. Some titles of the movies are listed in Tab. 1. In total we had 36 movies.

In order to assess the hedonic and eudaimonic qualities of the movie watching experience we adapted the instrument proposed

\footnotetext{
${ }^{1}$ the movies were retrieved from the source http://www.imdb.com/search/title?year= $2016 \&$ title_type $=$ feature $\&$ and sorted by popularity
}

by [9]. For each movie, chosen as liked, the subjects had to answer the following eudaimonic-related questions on an agreement scale from 1 to 7 :

- I liked the movie because it challenged my way of seeing the world.

- I liked the movie because it made me more reflective.

- I liked the movie because it focused on meaningful human conditions.

- I liked the movie because it made me think.

- I liked the movie because it was about people's search for greater understanding in life.

- I liked the movie because it conveyed a profound message.

and on the following hedonic-related questions on the same scale:

- I liked the movie because I had fun watching it.

- I liked the movie because it made me laugh.

- I liked the movie because it was entertaining.

- I liked the movie because it was happy and positive.

The single eudaimonic and hedonic qualities for each liked movieuser pair were calculated by averaging the scores of the individual questions.

For the disliked movies, we inverted the questions above into the form I did not like the movie because it did/was not .... Similarly, we aggregated these scores into single eudaimonic and hedonic scores for each disliked movie-user pair.

We further used the TIPI instrument to measure personality [4] in terms of the five factors openness, conscientiousness, extraversion, agreeableness and neuroticism. We used the $[1,5]$ scale.

We ran the study through Amazon Mechanical Turk. After removing subjects who did not pass a control question and removing outliers using the Mahalanobis distance we had the answers of 84 subjects ( $M=34.2$ years, $S D=9.5$ years, 29 females $)$.

\section{RESULTS}

\subsection{Eudaimonic and Hedonic Characteristics of Users}

The data we collected shows an interesting pattern. In the case of liked movies, the users liked movies with high hedonic quality, which was expected (see Fig. 1). However, users divided themselves into two clusters in terms of the eudaimonic qualities of the liked movies (see Fig. 2): some users liked movies with high eudaimonic qualities (scores $>3$ ) while some users liked movies with low eudaimonic qualities (scores $<3$ ), which is reflected in the bimodal shape of the histogram in Fig. 2. This interesting shape of the eudaimonic histogram indicates that the users can be clustered into pleasure-seekers (high hedonic and low eudaimonic quality) and meaning-seekers (high hedonic and high eudaimonic quality). We performed a k-means clustering over all the hedonic and eudaimonic variables. The clustering outcome shows two clusters of users, which are clearly separated by a threshold in the hedoniceudaimonic scores space, which can be seen in Fig. 3. 


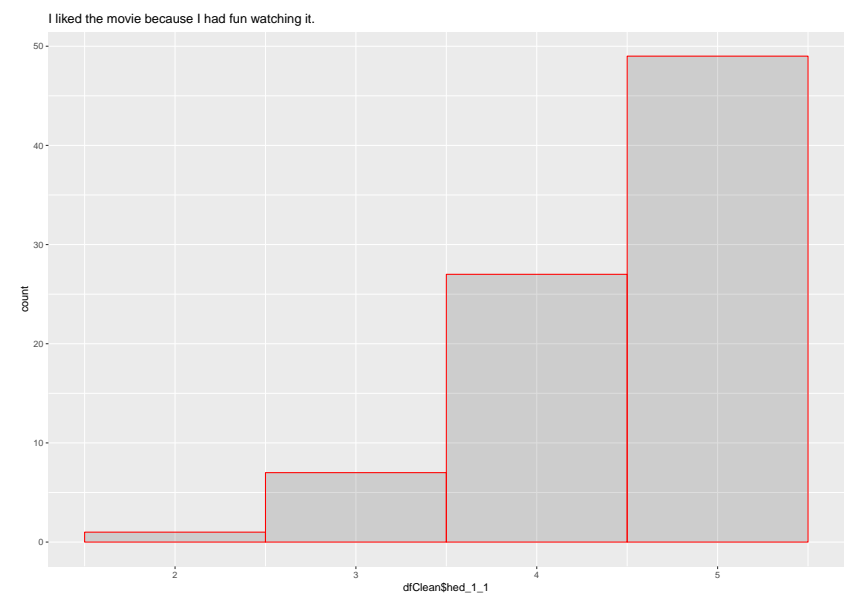

Figure 1: Distribution of hedonic qualities of liked movies. The variable reported in this figure is the agreement with the statement I liked this movie because I had fun watching it.

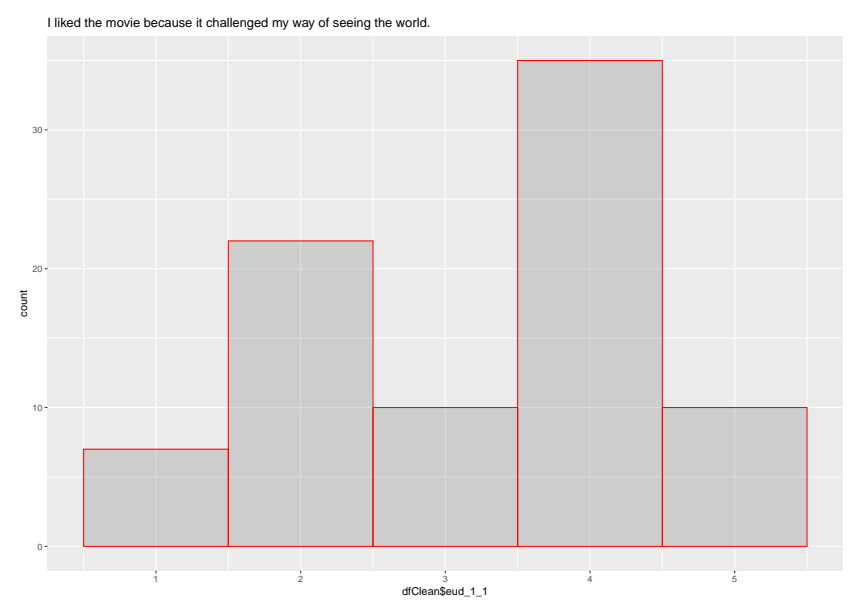

Figure 2: Distribution of eudaimonic qualities of liked movies. The variable reported in this figure is the agreement with the statement I liked this movie because it challenged my way of seeing the world.

\subsection{Eudaimonic and Hedonic Characteristics of Movies}

Here we cluster the movies, used in our study, into three clusters: (a) movies with predominantly eudaimonic quality, (b) movies with equal eudaimonic and hedonic qualities, and (c) movies with predominantly hedonic quality. In order to cluster the movies we perform the Wilcoxon rank sum test in order to test the hypothesis of the mean reported hedonic and eudaimonic quality being equal. Examples from all three clusters are reported in Tab. 2.

Beside the movies reported in Tab. 2, we observed strong differences in mean eudaimonic and hedonic qualities in some other movies, such as 12 Angry Men, and The Shawshank Redemption



Figure 3: Two clusters (blue:pleasure-seekers and red:meaning-seekers) were identified using k-means over all eudaimonic and hedonic variables.

Table 2: Examples from clusters of movies. The left column shows movies that have a stronger eudaimonic quality, the right column shows movies with a stronger hedonic quality and the mid column shows movies that are equally hedonic and eudaimonic

\begin{tabular}{lll}
\hline Eudaimonic & Mixed & Hedonic \\
\hline Arrival & La La Land & Deadpool \\
Passengers & Hidden Figures & Mad Max: Fury Road \\
The Girl on the Train & & Bad Moms \\
Fifty Shades of Gray & & \\
\hline
\end{tabular}

(higher eudaimonic quality) and Jurassic World and Baby Driver (higher hedonic quality). However, due to too little data at our disposal, the differences of means were not significant at $\alpha=0.05$.

\subsection{Personality Correlates with Eudaimonic and Hedonic Preferences}

We further analyze the relationships between user personality, and preferences for hedonic and eudaimonic content.

In our experiment, each subject picked a movie she liked and another she disliked. The correlations between personality factors in terms of the Five Factor Model end the chosen movie characteristics in terms of eudaimonic and hedonic preferences are presented separately for liked and disliked movies (Tab. 3). The personality factors were on the scale $[1,5]$ whereas the eudaimonic and hedonic values are on the scale $[1,7]$.

The data shows that there are significant correlations between openness, agreeableness, and neuroticism on one side and eudiamonic and hedonic qualities on the other side.

The questions for the eudaimonic/hedonic quality of the movies they liked (as reported in Sect 3) could be summarized as I like the movie because it has eudaimonic quality (the liked-eud column in Tab 3) and I like the movie because it has hedonic quality (the 
Table 3: Correlations between personality factors and eudaimonic/hedonic qualities of liked and disliked movies. The values in bold are significant correlations at 0.05

\begin{tabular}{lllll}
\hline & liked & \multicolumn{3}{l}{ disliked } \\
personality & eud & hed & eud & hed \\
\hline openness & 0.09 & $\mathbf{- 0 . 2 9}$ & $\mathbf{0 . 2 7}$ & 0.19 \\
conscientiousness & -0.19 & 0.13 & 0.14 & 0 \\
extraversion & 0.12 & -0.03 & 0.09 & -0.05 \\
agreeableness & 0.12 & 0.06 & $\mathbf{- 0 . 2 5}$ & -0.09 \\
neuroticism & $\mathbf{0 . 2 3}$ & -0.02 & -0.21 & 0.04 \\
\hline
\end{tabular}

liked-hed column in Tab 3). Hence, the positive correlation between neuroticism and liked-hed means that subjects who score high on neuroticism liked movies with high eudaimonic quality whereas those with low neuroticism liked movies with low eudaimonic quality. A similar pattern is observed for openness, but as a negative correlation to hedonic quality. In fact, subjects who scored high on openness liked movies with low hedonic quality whereas subjects with low openness liked movies with high hedonic qualities.

The questions for the eudaimonic/hedonic quality of the movies they disliked could be summarized as I did not like the movie because it did not have eudaimonic quality (the disliked-eud column in Tab 3) and I did not like the movie because it did not have hedonic quality (the disliked-hed column in Tab 3). Hence, the positive correlation between openness and disliked-eud indicates that people who scored high on openness did not like movies that did not contain eudaimonic quality. An opposite effect can be observed for people who score high on agreeableness. Agreeable people make it clear that the lack of eudaimonic quality is not the reason they don't like a movie, whereas non-agreeable people dislike movies because of the lack of eudaimonic content.

\section{LIMITATIONS AND FUTURE WORK}

Our study shows promising reults and we plan to carry on with research. The main limitations of our study are:

- small number of subjects

- intrusive assessment of user preferences through questionnaires

- intrusive assessment of the movies' qualities

While the first limitation can be addressed simply by extending the study to a larger sample, the other two require additional work.

The assessment of user preferences through questionnaires, such as the one used in this study, is too intrusive and cumbersome. Based on our prior work on inference of personality from social media $[2,11]$ we plan to adopt a similar approach to infer the propensity of user for eudaimonic and hedonic content from social media. We plan to train a model that takes as input features extracted from social media activity and make a prediction of the propensity of each user for eudaimonic and hedonic entertainment.

Similarly, inferring the quality of movies through extensive questionnaires is not feasible for a personalized system of large scale. Based on the work of Wirth et al. [13] we conjecture that movie content contains information pertaining to eudaimonic and hedonic qualities. In order to devise an automatic method we plan to train a predictive model that takes as input movies' subtitles and infers their eudaimonic and hedonic qualities. Feature engineering will be done using Natural Language Processing techniques.

\section{CONCLUSION}

In this short paper we present the outcomes of a preliminary study on the influence of eudaimonic and hedonic qualities of movies on user preferences.

The results of our study show that the variance in user preferences (in terms of eudaimonic and hedonic qualities) is reflected also in personality. A correlational analysis showed that people who score high on openness and neuroticism tend to prefer movies with more eudaimonic than hedonic qualities. Similarly, people high in openness dislike a movie if it does not contain eudaimonic qualities. The opposite effect was found for agreeableness.

Furthermore, we lay out a research plan for the following steps, which include: (i) unobtrusive inference of user preferences in terms of eudaimonic and hedonic qualities from social media, (ii) robust automatic inference of movie quality from subtitles and (iii) prediction of movie preference in the form of a recommender system.

\section{REFERENCES}

[1] Antonella Delle Fave, Fausto Massimini, and Marta Bassi. 2011. Hedonism and Eudaimonism in Positive Psychology. Springer Netherlands, Dordrecht, 3-18. https://doi.org/10.1007/978-90-481-9876-4_1

[2] Bruce Ferwerda, Markus Schedl, and Marko Tkalcic. 2015. Predicting Personality Traits with Instagram Pictures. In Proceedings of the 3rd Workshop on Emotions and Personality in Personalized Systems 2015 - EMPIRE '15, Marko Tkalčič, Berardina De Carolis, Marco de Gemmis, Ante Odić, and Andrej Košir (Eds.). ACM Press, New York, New York, USA, 7-10. https://doi.org/10.1145/2809643.2809644

[3] Bruce Ferwerda, Marko Tkalcic, and Markus Schedl. 2017. Personality Traits and Music Genres. In Proceedings of the 25th Conference on User Modeling, Adaptation and Personalization - UMAP '17. ACM Press, New York, New York, USA, 285-288. https://doi.org/10.1145/3079628.3079693

[4] Samuel D Gosling, Peter J Rentfrow, and William B Swann. 2003. A very brief measure of the Big-Five personality domains. Fournal of Research in Personality 37, 6 (dec 2003), 504-528. https://doi.org/10.1016/S0092-6566(03)00046-1

[5] Raghav Pavan Karumur, Tien T. Nguyen, and Joseph A. Konstan. 2016. Exploring the Value of Personality in Predicting Rating Behaviors. In Proceedings of the 10th ACM Conference on Recommender Systems - RecSys '16. ACM Press, New York, New York, USA, 139-142. https://doi.org/10.1145/2959100.2959140

[6] Feng Kong, Ling Liu, Xu Wang, Siyuan Hu, Yiying Song, and Jia Liu. 2015. Different neural pathways linking personality traits and eudaimonic well-being: a resting-state functional magnetic resonance imaging study. Cognitive, Affective, \& Behavioral Neuroscience 15, 2 (01 Jun 2015), 299-309. https://doi.org/10.3758/ s13415-014-0328-1

[7] Robert R McCrae and Oliver P John. 1992. An Introduction to the Five-Factor Model and its Applications. Fournal of Personality 60, 2 (1992), p175 - 215.

[8] Elisa D. Mekler and Kasper Hornbæk. 2016. Momentary Pleasure or Lasting Meaning?: Distinguishing Eudaimonic and Hedonic User Experiences. Proceedings of the 2016 CHI Conference on Human Factors in Computing Systems - CHI '16 (2016), 4509-4520. https://doi.org/10.1145/2858036.2858225

[9] Mary Beth Oliver and Arthur A. Raney. 2011. Entertainment as Pleasurable and Meaningful: Identifying Hedonic and Eudaimonic Motivations for Entertainment Consumption. Journal of Communication 61, 5 (2011), 984-1004. https://doi.org/ 10.1111/j.1460-2466.2011.01585.x

[10] Peter J. Rentfrow and Samuel D. Gosling. 2003. The do re mi's of everyday life: The structure and personality correlates of music preferences. Fournal of Personality and Social Psychology 84, 6 (2003), 1236-1256. https://doi.org/10. 1037/0022-3514.84.6.1236

[11] Marcin Skowron, Marko Tkalčič, Bruce Ferwerda, and Markus Schedl. 2016. Fusing Social Media Cues. In Proceedings of the 25th International Conference Companion on World Wide Web - WWW'16 Companion. ACM Press, New York, New York, USA, 107-108. https://doi.org/10.1145/2872518.2889368

[12] Marko Tkalčič, Bruce Ferwerda, David Hauger, and Markus Schedl. 2015. Personality Correlates for Digital Concert Program Notes. In UMAP 2015, Lecture Notes On Computer Science 9146. Vol. 9146. 364-369. https://doi.org/10.1007/ 978-3-319-20267-9_32 
[13] Werner Wirth, Matthias Hofer, and Holger Schramm. 2012. Beyond Pleasure: Exploring the Eudaimonic Entertainment Experience. Human Communication Research 38, 4 (2012), 406-428. https://doi.org/10.1111/j.1468-2958.2012.01434.x 